\title{
Fibre optic sensors for high speed hypervelocity impact studies and low velocity drop tests
}

\author{
D A Jackson*, Applied Optics Group, M J Cole*, M J Burchell*, Space Group and D J Webb \\ Photonics Research Group \\ *School of Physical Sciences, University of Kent, Canterbury, Kent, UK, CT2 7NH \\ ${ }^{\circ}$ Aston University, Aston Triangle, Birmingham, UK, B4 7ET
}

\begin{abstract}
The initial aim of this project was to develop a non-contact fibre optic based displacement sensor to operate in the harsh environment of a 'Light Gas Gun' (LGG), which can 'fire' small particles at velocities ranging from 1-8.4 km/s. The LGG is used extensively for research in aerospace to analyze the effects of high speed impacts on materials. Ideally the measurement should be made close to the centre of the impact to minimise corruption of the data from edge effects and survive the impact. A further requirement is that it should operate at a stand-off distance of $\sim 8 \mathrm{~cm}$. For these reasons we chose to develop a pseudo con-focal intensity sensor, which demonstrated resolution comparable with conventional PVDF sensors combined with high survivability and low cost. A second sensor was developed based on 'Fibre Bragg Gratings' (FBG) which although requiring contact with the target the low weight and very small contact area had minimal effect on the dynamics of the target. The FBG was mounted either on the surface of the target or tangentially between a fixed location. The output signals from the FBG were interrogated in time by a new method. Measurements were made on composite and aluminium plates in the LGG and on low speed drop tests. The particle momentum for the drop tests was chosen to be similar to that of the particles used in the LGG.
\end{abstract}

Keywords: intensity sensor, FBG, hypervelocity impacts, composite, high speed processing

\section{INTRODUCTION}

The Kent two stage LGG Fig 1 fires small projectiles (microns to $\mathrm{mm}$ size) at speeds from 1 to $8.4 \mathrm{~km} / \mathrm{s}$. The speed is measured in each shot to better than $1 \%$ accuracy and the target chamber evacuated to typically 0.5 mbar during a shot. Targets can be powered and instrumented during a shot, permitting real time read out of any impact sensors on the target. The aim of the project was to develop robust fibre optic based sensors to monitor the damage caused by very high speed impacts.

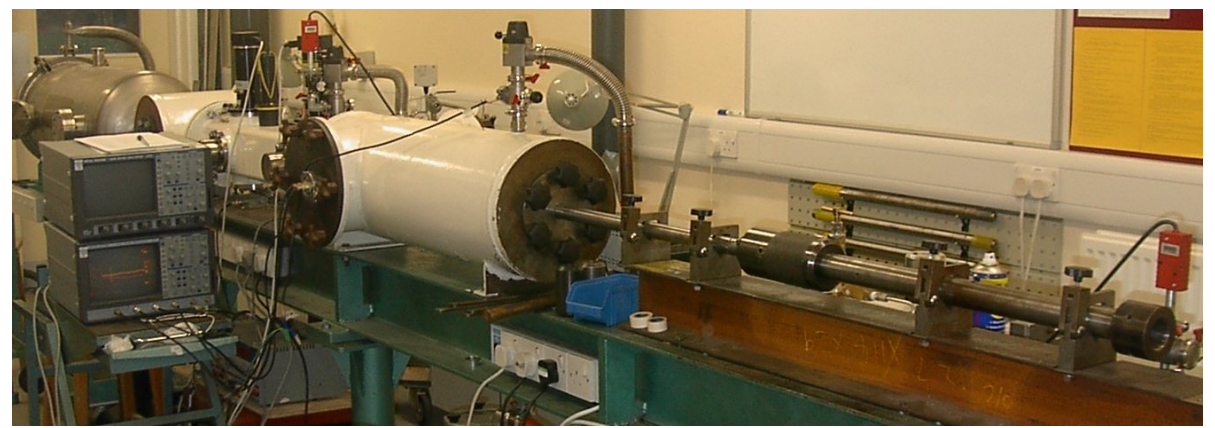

Fig 1 Light Gas Gun

The presence of cosmic dust in space, combined with the growth of man-made debris in some Earth Orbits, means that space vehicles are subject to frequent impacts by small high speed objects during their lifetime in space. Equipping space vehicles with impact sensors helps monitor the growth of this dust flux. Further, real time readout of impact sensors with diagnostic capabilities (e.g. energy or momentum of impact, location of impact on a larger surface etc.) is potentially a vital tool in monitoring damage to long term space assets such as the \$100bn International Space Station. 


\section{INTENSITY SENSOR}

The Intensity sensor is shown schematically as it would be aligned with the target fig 2 . Light from a broad band source at $1.50 \mu \mathrm{m}$ is injected into a single mode fibre and transferred by a circulator to an adjustable collimator with an NA 0.53

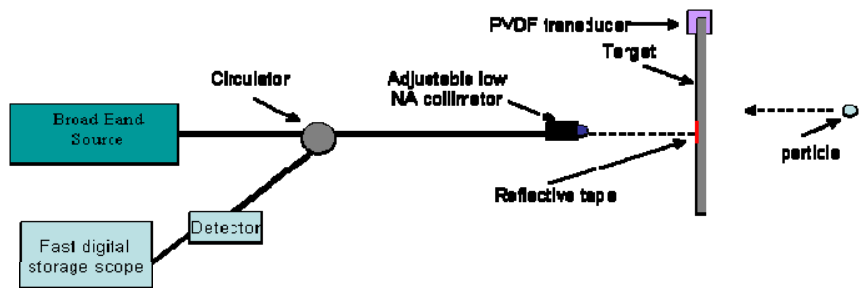

Fig.2 Optical topology of intensity sensor aligned with the target

and back reflected from $3 \mathrm{M}$ reflective tape mounted on the target, this signal is transferred to a high sensitivity detector and stored in a fast digital 'scope', operated in a single shot mode and triggered by a pulse generated by the ' particle' passing through a laser gate set across the particle flight path. A signal is also derived from a PVDF transducer mounted on the side of the target and stored in the $2^{\text {nd }}$ channel of the 'scope' such that the PVDF and optical signals are recorded simultaneously after the trigger. Ideally the reflective tape should be placed directly in the region of the impact; unfortunately this is not possible as it would be destroyed in the impact, it was therefore placed $\sim 1.5 \mathrm{~cm}$ away from the impact location. Although information is lost this approach in principle it provides the most accurate information on the dynamics of the impact which is not the case of the PVDF as it's mounted on the edge of the target. To protect the optics a Perspex screen was interposed between the collimator and the target. The collimator was mounted $\sim 8 \mathrm{~cm}$ away from the target and tightly focussed on the tape to maximize the back reflected optical signal (I max). The angular orientation of the collimator was set such that the recovered optical power was Imax/2 to ensure a symmetrical intensity signal. Figures 3a shows, intensity sensor 'scope' traces for a $1.966 \mathrm{~km} / \mathrm{s}$ particle, fig $3 \mathrm{~b}$ and $3 \mathrm{c}$ scope traces transferred for analysis for a $3.94 \mathrm{~km} / \mathrm{s}$ particle.

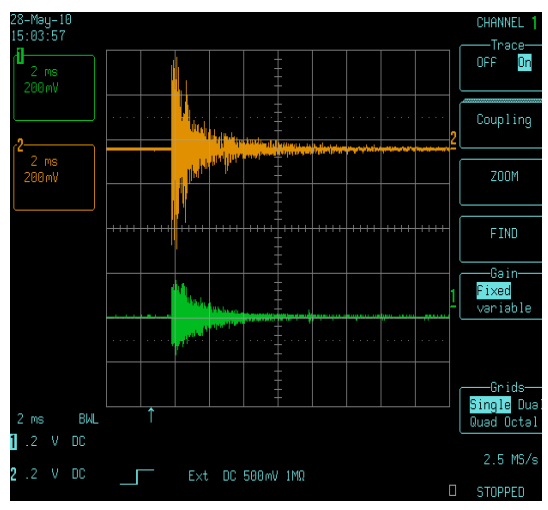

Fig 3a Intensity sensor, top trace and PVDF sensor lower trace, $1.966 \mathrm{~km} / \mathrm{s}$, particle $1 \mathrm{~mm}$ stainless steel

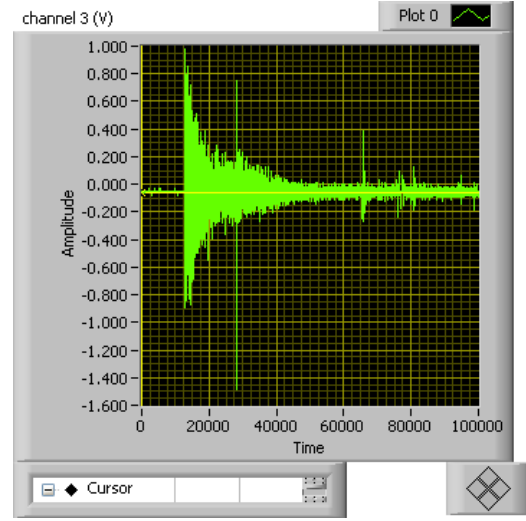

Fig $3 \mathrm{~b}$ Intensity sensor trace $3.94 \mathrm{~km} / \mathrm{s}$ particle, $1 \mathrm{~mm}$ stainless steel

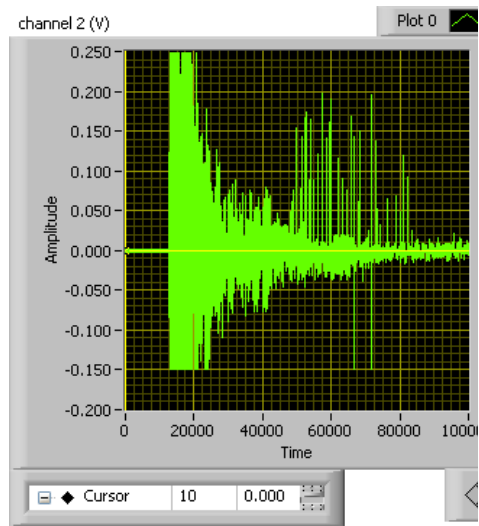

Fig 3c PVDF sensor trace, 3.94

$\mathrm{km} / \mathrm{s}$, particle $1 \mathrm{~mm}$ stainless steel

\section{CALIBRATION AND SENSITIVITY}

For 1 shot at $3.94 \mathrm{~km} / \mathrm{s}$, the measured movement of the back of target plate had an estimated maximum speed of $130 \mathrm{~m} / \mathrm{s}$ with peak to peak oscillation amplitude of $260 \mu \mathrm{m}$, for a second shot at $1.069 \mathrm{~km} / \mathrm{s}$ the maximum speed of the back surface was $18 \mathrm{~m} / \mathrm{s}$ with peak to peak amplitude of $42 \mu \mathrm{m}$. With the measured noise of $+/-9.4 \mathrm{mV}$, around $3 \mu \mathrm{m}$ movements can be seen at the 3 sigma level of detection. 


\section{MOUNTING THE FBG ON THE TARGET PLATES}

The targets evaluated for impact damage were aluminium or composite plates $1.6 \mathrm{~mm}$ and $1.5 \mathrm{~mm}$ thick respectively, the plates were $15 * 15 \mathrm{~cm}$ with mounting holes at each corner. The plates were mounted on a free standing rigid jig designed for drop tests or mounting in the LGG. The Jig allowed the FBG to be either surface or orthogonally mounted to the plate. For surface mounting the FBG was attached at $\sim 1.5 \mathrm{~cm}$ from the centre of the plate along a line parallel to the plate edge. In the mounting process the FBG was linearly strained to ensure bidirectional strain response (SUFBG). To enable orthogonal mounting a small hole was drilled through the plate $\sim 2.5 \mathrm{~cm}$ from the plate centre. Part of the input fibre just before the FBG was attached to a miniature 3D translation stage. The fibre on the other side of the FBG was passed through the small hole on the surface of the plate and attached to a small fitting. The FBG was then statically strained at $\sim 2$ millistrain again ensuring bidirectional strain response (OTFBG). Stainless steel bearings of 8.31 or $32.57 \mathrm{~g}$ were used for the drop tests. The drop height defined as the distance between the drop-point and the top surface of the plate gave the particle momentum. The heights used were 172 and $169 \mathrm{~mm}$, respectively. The distance between the translator stage attachment point was $6.4 \mathrm{~cm}$.

\section{INTERROGATION}

The stress induced change in the mean reflecting wavelength of the FBG was converted to the time domain as described by Jackson ${ }^{1}$.This method has the ability to measure high strain at high frequencies. Although in ref. 1 the FBG signals were converted to pulses this method would allow the dynamic variation of the spectral profile of the FBG to be determined during an impact as recently reported by Propst ${ }^{2}$. For the measurements reported here the system scanning rate was $5 \mathrm{kHz}$, the number of sequences in the water fall was 500 and the time between sequences $200 \mu \mathrm{s}$.

\section{RESULTS}
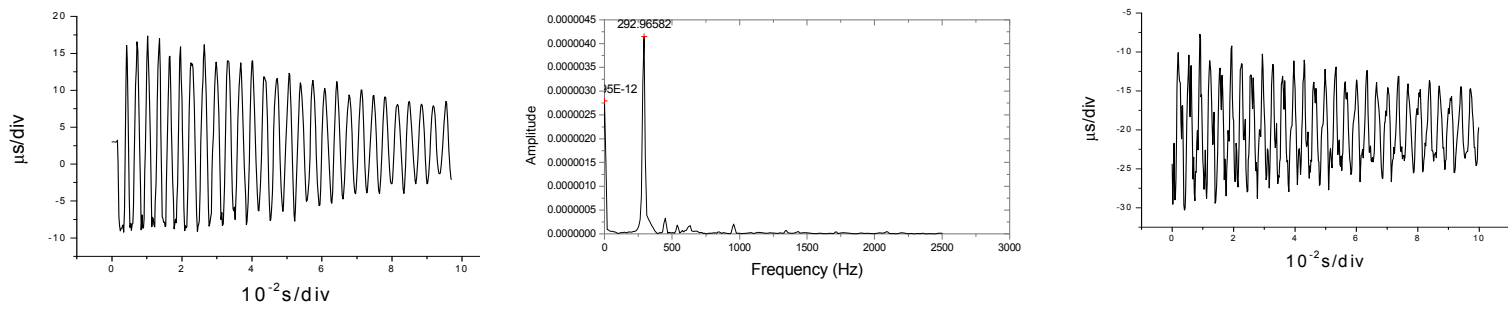

Fig 4a Composite plate OTFBG

Fig $4 b$ FFT of results of fig $4 a$

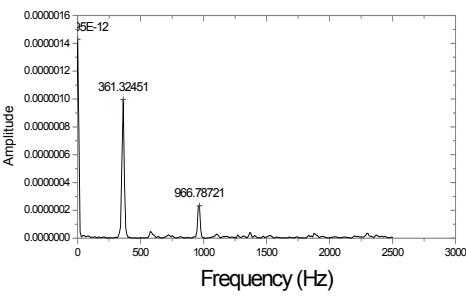

Fig5b FFT of results of fig 5a
Fig 4c Composite plate SUFBG

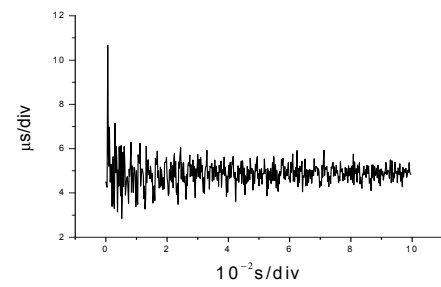

Fig 5c Aluminium plate SUFBG

Fig 5a Aluminium plate OTFBG

Sec; 6.1; Drop tests: To ensure consistent results for the drop tests an optical gate was incorporated into the rig, which triggered when the particle passed through it and initiated data collection. Fig. 4a shows the output signal from an OTFBG on a composite plate, fig. $4 \mathrm{~b}$ shows its FFT with a dominate peak at $292.9 \mathrm{~Hz}$. The maximum time deviation of the FBG caused by the impact can be converted to strain using $\Delta \mathrm{t} / \Delta \varepsilon=3.52 \pm 0.13 \mu \mathrm{s} / 1000 \mu \mathrm{strain}$ ref. ${ }^{1}$ The induced strain in the OTFBG is used to determine the out of plane displacement of the plate which was $0.436 \mathrm{~mm}$. Fig $4 \mathrm{c}$ shows the 
trace for a SUFBG, the FFT of this trace had a weak peak at $292 \mathrm{~Hz}$. The surface strain of the composite plate SUFBG was $5680 \mu$ strain determined from the maximum time deviation. Similar experiments were performed with the aluminium plate which for an OTFBG had a peak at $361.3 \mathrm{~Hz}$ in the FFT. The out of plane displacement was $0.21 \mathrm{~mm}$.

Sec; 6.2 LGG Experiments on the LGG were performed on composite and aluminium plates using the same OTFBG and SUFBG configurations as the drop test. The traces in fig $6 \mathrm{a}$ and $6 \mathrm{~b}$ are for a composite with OTFBG, trace $6 \mathrm{a}$ is the impact induced FBG signal, $6 \mathrm{~b}$ its FFT which shows a strong peak at $312.5 \mathrm{~Hz}$. Traces $7 \mathrm{a}$ and $7 \mathrm{~b}$ shows very complex changes in the induced strain of the FBG and its spectrum. SUFBG traces $6 \mathrm{c}$ and $7 \mathrm{c}$ contain data that cannot be readily processed and is beyond the scope of this project. The out of plane displacement derived from fig $6 \mathrm{a}$ was $0.46 \mathrm{~mm}$.

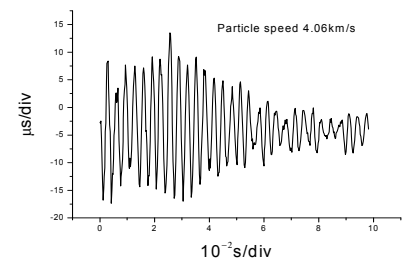

Fig $6 \mathrm{a}$

Composite OTFBG

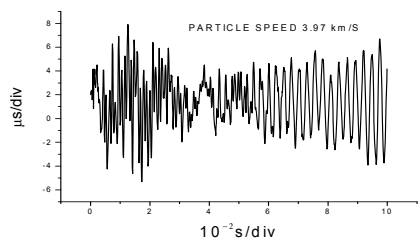

Fig 7a Aluminium OTFBG

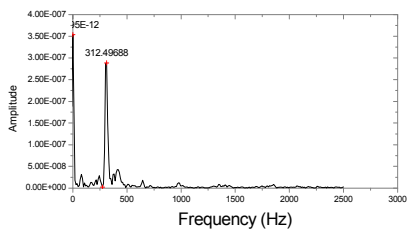

Fig $6 \mathrm{~b} \quad$ FFT of results of fig $6 \mathrm{a}$

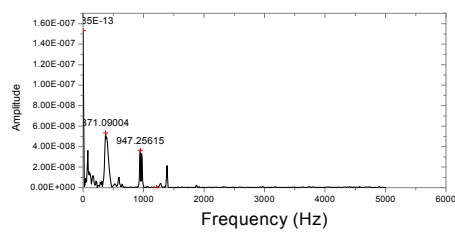

Fig $7 \mathrm{~b} \quad$ FFT of results of fig $7 \mathrm{a}$

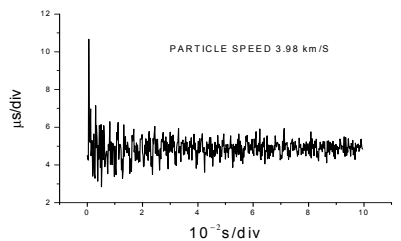

Fig 6c Composite SUFBG

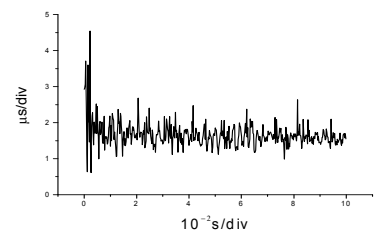

Fig 7c Aluminium SUFBG

\section{COMMENTS}

The intensity sensor target displacement was determined by an ancillary measurement where the target mounted on a linear scanning translation stage was driven through the intensity profile of the collimator focal profile, the minimum detectable displacement was 3um. The drop tests showed that for low momentum drops high quality signals could be recovered with the OTFBG configuration whereas the result from the SUFBG were poor. The main reason is that structural tests would normally be performed with much larger masses ${ }^{2}$. In fact excellent signals could be obtained by simply bending the plates manually. The signals obtained with the LGG, (OTFBG) look promising with the composite providing some useful results whereas the results with SUFBG are not encouraging. In the case of the drop test the minimum detectable displacement with the OTFBG was $1.24 \mu \mathrm{m}$, the sensitivity could be improved by using a shorter fibre. The OTFBG mounted on the composite plate gives a minimum momentum detection of $4.4 \times 10^{-5} \mathrm{Ns}$ while the aluminium gives only $1.06 \times 10^{-4} \mathrm{Ns}$. The situation is reversed for the SUFBG arrangement where the aluminium is sensitive down to $9.7 \times 10^{-4} \mathrm{Ns}$; the composite sensitivity is $\sim 1.0 \times 10^{-3} \mathrm{Ns}$, probably because the composite stiffness works against transmitting a bending wave that could stretch the fibre. Despite the hazardous tests the FBG suffered none were broken!

\section{ACKNOWLEDGEMENTS}

The authors gratefully acknowledge Dr Luis Ferreira of Fibre Sensing (Portugal) for supplying the FBG and Dr John Nixon of Advanced Composite Group Ltd (UK) for supply the composite samples.

\section{REFERENCES}

1. Jackson, D, A., "Fibre Bragg Gratings Subject to High Strain at High Frequencies", submitted to OFS 212010

2. Propst, A., Peters, K., Zirkry, M.A., Kunzler, W., Zhu, Z., Wirthlin, N., Selfidge, R., Schultz, S.," Dynamic, fullspectral interrogation of fiber bragg grating sensors for impact testing of composite laminates." OFS20 SPIE vol.7503, $75030 \mathrm{G}, 2009$ 